\title{
Semiconductor Materials for Fabrication of Multi-Color Organic Light-Emitting Diodes (OLEDs)
}

\author{
Aftab Ahmad ${ }^{1}$, Nasif Raza Jaffri, ${ }^{1,2 a}$, Usama Abrar ${ }^{1,2 b}$ \\ RECEIVED ON 23.05.2019, ACCEPTED ON 05.05.2021
}

\begin{abstract}
This study aims to propose organic materials for the development of light-emitting semiconductor diodes for colored displays. Studies show that these materials are capable of creating a variety of different colors rather than white light. But of Organic Light-Emitting Diodes (OLEDs) are only used as a source of white back-light for OLED displays and liquid crystals are used for color generation. This work suggests that OLEDs can be used to make color displays on their own without the help of Liquid Crystals (LCs). Recently, organic devices are widely under discussion as are comparatively cheap, can be processed economically and effortlessly at ambient temperature besides their effortless handling. The calculation of the electronic properties of molecular species was achieved by the use of ab-initio quantum mechanical methods, i.e., Density Functional Theory (DFT). DFT methods are suited to calculate the electronics properties of the organic molecules, enabling the determination of band gaps and quantum efficiencies. DFT views electron stochastic nature and thus calculates the material's solid-state properties. DFT calculations on isolated molecules were carried by the Gaussian software package to predict electronic properties. Pentacene is used as test molecule in this work. B3LYP functional use Kohn-Sham orbitals to predict the band energy values said material rather than LDA functional that depends on the value of electronic density at each point on the space. The substitution process was used to make changes in bandgaps; which affect shades of light emitted by OLEDs.
\end{abstract}

Keywords: Organic Semiconductor for LED, Organic Semiconductor, Organic LED, OLED

\section{INTRODUCTION}

$\mathrm{F}$ or the last 50 years Silicon has been the major raw material in electronics manufacturing [1,

2]. Electronic properties of organic solids were first noticed in the 1960s [3]. At that time only a basic understanding of the materials was known, mainly focusing on one component of organic molecular crystals [2, 3]. However, it was soon concluded that like inorganic materials, roots of electrons and holes can be defined in organic materials as the charge organic materials as the charge carrier band is narrow in this case [4]. An Organic material with $\pi$-bond
(Fig.1) contains electronics properties [5]. When the bonds are not between the nuclei and form cloud above, such bonds are known as $\pi$-bonds [6]. This type of bond is normally present in double and triple covalent bonds and it is not in-between nuclei indicating that this bond possesses high energy with loosely bonded [5]. Electrons make clouds over the nucleus, which helps them to jump from one atom to another. These features are good for the free movement of electrons and electronic conductivity.

The efficiency of the organic devices depends upon the charge injection and chances of charge capture or maintaining balance in the injection of electrons from

\footnotetext{
${ }^{1}$ Department of Electrical Engineering, NFC-Institute of Engineering and Fertilizers Research, Faisalabad, Pakistan. Email: aftabahamadrao@gmail.com

${ }^{2}$ School of Control and Computer Engineering, North China Electric Power University Beijing, 102206 China. Email: nasifraza@hotmail.com, busamaabrar@gmail.com
}

This is an open access article published by Mehran University of Engineering and Technology, Jamshoro under CC BY 4.0 International License. 


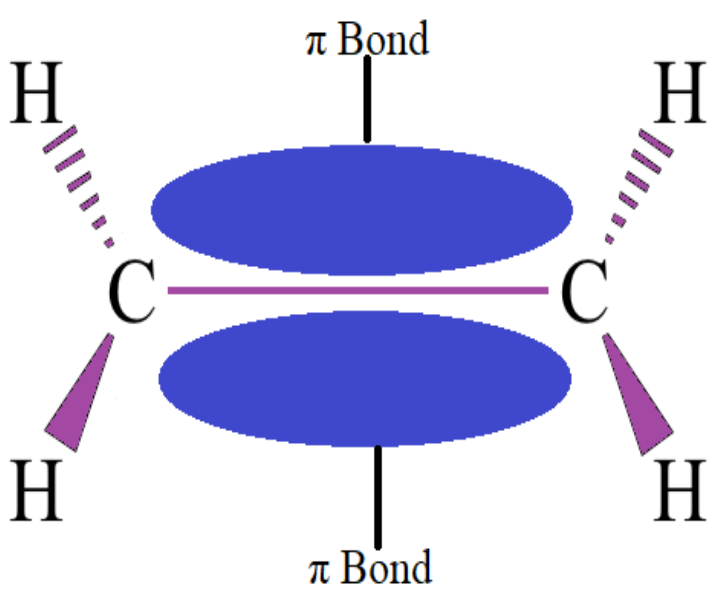

Fig. 1: $\pi$-bond formation

one side and holes from another side. The device (OLED) is placed in between two electrodes, the negative called as Lowest Unoccupied Molecular Orbital (LUMO) and positive as Highest Occupied Molecular Obital (HOMO) [7].

\subsection{Organic Light Emitting Diode (OLED)}

This paper is based on the organic light-emitting diode and will show how organic material can produce different colors out of it. These light-emitting diodes are thin, smart, bright, low cost, and low power [8]. Moreover, organic light-emitting diodes do not suffer from viewing angle limitations, which is their biggest advantage over Liquid Crystal (LC) displays $[9,10]$. Because of these features, they could be widely used in a variety of displays. Optoelectronics of organic materials is very much in its infancy, and the subject of research during the last two decades but it still faces some difficulties and has not therefore been reached any major commercial development [11].

In OLEDs, organic semiconductor is sandwiched between two electrodes. However, the effect of diffusion being small can be neglected [12]. The relationship between the current density $\mathrm{J}$ and applied bias $\mathrm{V}$ is given by the equation

$\mathrm{J}=\frac{9}{8} \epsilon_{\mathrm{o}} \epsilon_{\mathrm{r}} \mu \frac{\mathrm{V}^{2}}{\mathrm{~L}^{3}}$

Where $\epsilon_{\mathrm{r}}$ is medium dielectric constant and $\mathrm{L}$ is the thickness of the device.

Fig. 2 shows the structure of OLED. Normally, two different types of organic materials are used, one is used for the emissive layer and the other for the conductive layer [8]. The emissive layer is attached to the cathode and transport electrons from the cathode. While the conductive layer is attached to the anode and transport holes [11]. When electrons in the emissive layer try to fill holes in the conductive layer, an extra bit of energy is released in the form of the photon which emits light. All of the structure is put onto a flexible transparent substrate by which omission of light can be observed.

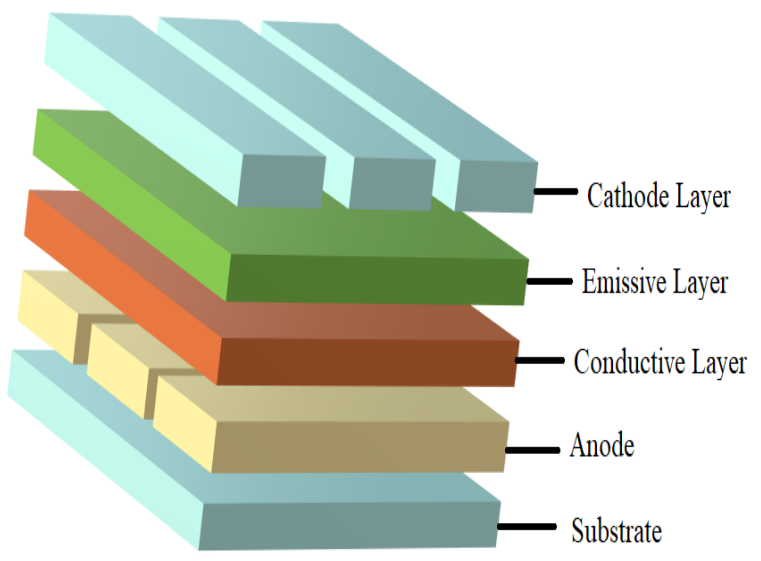

Fig. 2: OLED Structure

\subsection{Pentacene}

In this paper organic material under study is Pentacene. To term a selection of organic material a good selection, it must have the ability to be easily fabricated on a flexible substrate with cheap printing techniques along-with better motilities. Pentacene has all these properties. Based on these properties if one selects Pentacene for OFETS or OLEDs, it would not be a bad choice.

This is found in powder form and dark blue color. Pentacene shows sensitivity to light. The organic phototransistors (PTs) show high mobility of $0.4^{2} \mathrm{~V}^{1} \mathrm{~s}$ ${ }^{1} \mathrm{~cm}$, a maximum photoresponsivity of about 1000 $\mathrm{A} / \mathrm{W}$, and a photocurrent/dark-current ratio of around 800 under white light irradiation with $30 \mu \mathrm{W} / \mathrm{cm}^{2}$. The data are comparable with those of PTs based on amorphous silicon. Meanwhile, it is interesting to see that the devices show a high air-stable property and high photosensitivity via an electric-field enhanced process. All these phenomena were attributed to the 
intrinsic optic-electronic property of the organic semiconductor and optic-electric field effect [13].

Pentacene was among the first listed organic material which shows electronic properties in it. The mobility of holes in Pentacene is higher as compared to other organic materials. It tends to form well-ordered molecular crystals. Pentacene has emerged as an attractive candidate for fabricating p-channel Organic Thin-Film Transistors (OTFTs). It is one of the few known p-channel organic semiconductors that regularly exhibit mobilities over $1 \mathrm{~cm}^{2} / \mathrm{Vs}$ on $\mathrm{SiO}_{2}$. The transistors sometimes exhibit mobilities over 1 $\mathrm{cm}^{2} / \mathrm{Vs}[13]$.

\section{METHODOLOGY}

To make any color display, it is needed to produce three basic colors (red, blue, and green/RGB) [14]. An important factor in this regard is the tuning of the organic materials which have to be used for organic light-emitting diodes manufacturing. Making a little change in the materials can change their HOMO and LUMO levels. By varying HOMO and LUMO levels, different colors of light can be emitted through the organic light-emitting diodes. This paper deals with the changes in HOMO/LUMO level by substituting different materials in it and hence the production of different colors using OLED [15] compare to Prabu et. al., [16] used the substitution only for the investigation of vibrational modes.

The discovery of $\pi$-conjugate in organic materials has been increased the rate of research and study. Organic semiconductors are considered as the better choice for large-area flexible displays and LED's and solar cells [17]. The performance of these devices depends upon the efficiency of charge carriers in organic $\pi$ conjugated materials, these charge carriers can be injected with the help of conducting metal or oxide electrodes attached to semiconductors in case of OFET and OLED or can be generated in solar cell's case [18].

Density Functional Theory is used to calculate the properties of materials under study. Mickenset. al., [19] used Thomas Fermi's level to calculate material properties but is not accurate as DFT [20]. Fu et. al., [21] Hartree-Fock method uses electron wave function for calculations, this createsa problem and makes calculate difficult for complex and large systems. On the other hand, DFT as the name suggests that this is the theory of density and functions, it uses electron charge density to make calculations [22]. Because of precise and fast results, DFT is considered one of the preferred systems for material properties calculations. DFT is based on the Hohenberg-Kohn theorem, this theorem defines the relationship between ground state electrons density and energy of the system.

There are two statements of these theorems; we will discuss each by one each and their mathematical derivations.

1. The two systems of different potentials $v_{2}(r)$ can have same ground state energy $n(r)$ only if the difference in these two potentials is equal to the constant.

The ground state material determines the properties of the system. By this HohenbergKohn can be defined in the universal function.

$$
\begin{aligned}
& \mathrm{F}[\mathrm{n}]=K[\mathrm{n}]+\mathrm{W}_{\mathrm{e}}[\mathrm{n}] \\
& \mathrm{F}[\mathrm{n}]=\mathrm{K}_{\mathrm{s}}[\mathrm{n}]+\frac{1}{2} \int \mathrm{d}^{3} \mathrm{rd}^{3} \mathrm{r}^{\prime} \frac{\mathrm{n}(\mathrm{r}) \mathrm{n}\left(\mathrm{r}^{\prime}\right)}{\left|\mathrm{r}-\mathrm{r}^{\prime}\right|}+E_{\mathrm{xc}}[\mathrm{n}]
\end{aligned}
$$

The term $K_{s}[n]$ in equation (2) is kinetic energy produced because of the electron gas, and is the same for both systems as the ground state energy of the systems is the same. The second term is electron-electron attraction and it is defining in form of a coulomb attraction formula. The last term in equation (2) is the correlation and the exchange energy [23].

2. As mentioned earlier, the ground state is used to determine the properties of the system. The second statement of the Hohenberg-Kohn theorem is about the ground state and is stated as functional that represents the ground state energy of the system. It represents the lowest energy if and only if the input density is ground state density [24].

$$
\mathrm{E}[\mathrm{n}]=\mathrm{F}[\mathrm{n}]+\int \mathrm{d}^{3} \mathrm{rV}_{\text {ext }}(\mathrm{r}) \mathrm{n}(\mathrm{r})
$$


The equation above, which defines the ground state energy of the system, has a few noticeable things in it. The ground state energy determines the external potential $V_{\text {ext }}$ and the smallest possible value of functional can only be obtained in a case where input is the pure ground state.

There is no color in the world; all the colors we see are an illusion created by the interactions of countless neurons present in our brain (Fig. 3). Hence these colors are produced by neural programs and projected into our brain. Energy and wavelength are the two properties of light that are responsible for this projection [25]. This means that varying wavelengths to produce a different color of emission is very important. This can be done by doping different materials at different positions in the chains to produce a result.

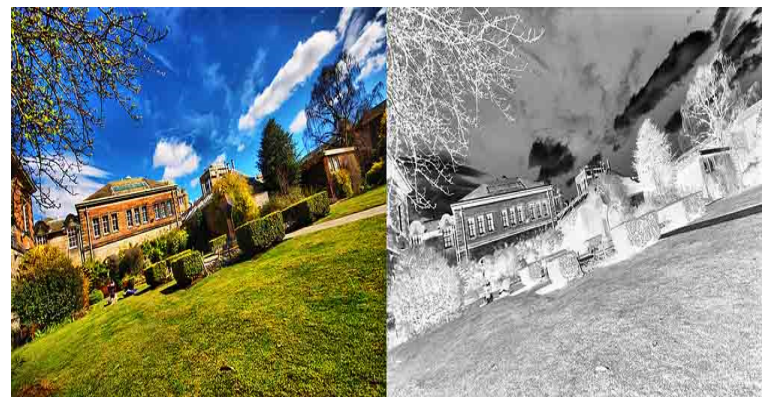

Fig. 3: How we see the world and the actual world

There are two different ways to use an organic lightemitting diode for displays: (i) Using white color OLED as a backlight, (ii) Producing RGB color from OLED to create an image. RGB stands for red, green, and blue. These three colors are basic; with the combination of these three colors, we can produce every color. The spectrum of light that is visible to the human eye is $380 \mathrm{~nm}$ to $700 \mathrm{~nm}$ [26].

The light with a short wavelength has pigment absorbing maximum at around $420 \mathrm{~nm}$ with blue color dominant, medium wavelengths at $530 \mathrm{~nm}$ with dominant green color, and large wavelength at $600 \mathrm{~nm}$ with dominant red color [27]. The ranges of wavelength and frequency to color produced are represented in Table 1 [26].

In this work, the second approach is used where three colors were omitted to produce an image from it. The wavelength of the Pentacene is varied by substituting single or double Fluorine, Bromine, and Nitrate at different positions to the understanding tuning of organic material [28].

Table 1: Light Wavelengths and Frequency by Color

\begin{tabular}{|c|c|c|}
\hline $\begin{array}{c}\text { Wavelength } \\
\text { (nm) }\end{array}$ & $\begin{array}{c}\text { Frequency } \\
\text { (THz) }\end{array}$ & Color \\
\hline $380-450$ & $668-789$ & Violet \\
\hline $450-495$ & $606-668$ & Blue \\
\hline $495-570$ & $526-606$ & Green \\
\hline $570-590$ & $508-526$ & Yellow \\
\hline $590-620$ & $484-508$ & Orange \\
\hline $620-750$ & $400-484$ & Red \\
\hline
\end{tabular}

\section{RESULTS AND DISCUSSION}

The Gaussian Software package is used for the calculations. The main calculations which were emphasized include HOMO and LUMO levels. With help of these values, energy bands were calculated which were further converted into wavelengths. The wavelength defines the omission of light color from the OLED. To predicts energies, there is a variety of functionals are present i.e., localization density approximation (LDA), B3LYP, sX-LDA. The first one is local next is hybrid and the last is non-local functional. For the local functional (LDA) electronic density only depends on the density value at each point in space. On the other hand, for hybrid (B3LYP) the exact exchange functional is expressed in terms of the Kohn-Sham orbitals [29]. The B3LYP is exchangecorrelation energy foundational in DFT. B3LYP has an exact exchange and correlation as compared to the fundamental basis set named LDA nut provides less electronic information as done by Borisov et. al.,[30] for only electrophilic calculation.

Gaussian Software Package needed inputs where coordinates of each atom defined. To calculate the coordinates of Pentacene, Autosketch version 15 was used. The drawing is shown in Fig. 4. The coordinates for each point are given in Table 3 . The bond length $1.4 \AA$ for $\mathrm{C}-\mathrm{C}$ bond while it is $1.08 \AA$ for $\mathrm{C}-\mathrm{H}$ bond. By using these values, the figure was drawn. However, it is noticeable that the coordinates of Fig. 4 shown in Table 3 have only two $x$ and $y$-axis. This suggests that 
the figure is two-dimensional. Gaussian Software Package is used to calculate properties of the single point molecule. For a single-point molecule, the size of the molecule is normally too small that its third coordinates can be neglected. Because of this reason the third coordinate is taken as 0 . The $\mathrm{C}-\mathrm{C}$ bond in Fig. 4 is drawn with red color while $\mathrm{C}-\mathrm{H}$ with blue. The basis set, used for the calculations, was B3LYP.

The said simulations were executed on the problem of Fig. 4 using Intel(R) Core(TM) i5-4300U CPU @ 1.9 $\mathrm{GHz} 2.5 \mathrm{GHz}$ with $4 \mathrm{~GB}$ RAM.The time taken by a processor with reasonable memory was about 11 hours. The band-gap calculated by HOMO and LUMO level is shown in Table 2. In Table 2, there are three values for the highest occupied orbital for the
Pentacene, amongst the highest is grey. The lowest unoccupied value is also in grey. By subtracting this value, the band-gap energy for Pentacene at ground state is calculated as 0.05097 Hartree energy. By multiplying this value with factor 27.211, this energy is converted into ev and is $1.38694467 \mathrm{ev}$.<smiles>CC1C(C)C(C)C2C(C)C3C(C)C4C(C)C5C(C)C(C)C(C)C(C)C5C(C)C4C(C)C3C(C)C2C1C</smiles>

Fig. 4: Structure of Pentacene

\begin{tabular}{|c|c|c|c|c|c|}
\hline Table 2: & Pentacene HOMO and LUMO \\
\hline \multicolumn{7}{|c|}{ Pentacene } \\
\hline Alpha occupied eigenvalues & -0.2148 & -0.20747 & -0.1583 & & \\
\hline Alpha unoccupied eigenvalues & -0.1074 & -0.04355 & -0.0402 & 0.0033 & 0.009 \\
\hline GAP HOMO/LUMO & 0.05097 & (Unit of gap is H) & & \\
\hline
\end{tabular}

\begin{tabular}{|c|c|c|c|c|c|c|c|c|}
\hline \multicolumn{8}{|c|}{ Table 3: Coordinates of Pentacene Structure } \\
\hline Point & $\mathbf{x}$ & $\mathbf{y}$ & Point & $\mathbf{x}$ & $\mathbf{y}$ & Point & $\mathbf{x}$ & $\mathbf{y}$ \\
\hline $\mathbf{1}$ & 0 & 1.4 & $\mathbf{2}$ & 1.2125 & 0.7 & $\mathbf{3}$ & 2.425 & 1.4 \\
\hline $\mathbf{4}$ & 3.6375 & 0.7 & $\mathbf{5}$ & 4.85 & 1.4 & $\mathbf{6}$ & 6.0625 & 0.7 \\
\hline $\mathbf{7}$ & 6.0625 & -0.7 & $\mathbf{8}$ & 4.85 & -1.4 & $\mathbf{9}$ & 3.6375 & -0.7 \\
\hline $\mathbf{1 0}$ & 2.425 & -1.4 & $\mathbf{1 1}$ & 1.2125 & -0.7 & $\mathbf{1 2}$ & 0 & -1.4 \\
\hline $\mathbf{1 3}$ & -1.2125 & -0.7 & $\mathbf{1 4}$ & -2.425 & -1.4 & $\mathbf{1 5}$ & -3.6375 & -0.7 \\
\hline $\mathbf{1 6}$ & -4.85 & -1.4 & $\mathbf{1 7}$ & -6.0625 & -0.7 & $\mathbf{1 8}$ & -6.0625 & 0.7 \\
\hline $\mathbf{1 9}$ & -4.85 & 1.4 & $\mathbf{2 0}$ & -3.6375 & 0.7 & $\mathbf{2 1}$ & -2.425 & 1.4 \\
\hline $\mathbf{2 2}$ & -1.2125 & 0.7 & $\mathbf{2 3}$ & 0 & 2.48 & $\mathbf{2 4}$ & 2.425 & 2.48 \\
\hline $\mathbf{2 5}$ & 4.85 & 2.48 & $\mathbf{2 6}$ & 6.998 & 1.24 & $\mathbf{2 7}$ & 6.998 & -1.24 \\
\hline $\mathbf{2 8}$ & 4.85 & -2.48 & $\mathbf{2 9}$ & 2.425 & -2.48 & $\mathbf{3 0}$ & 0 & -2.48 \\
\hline $\mathbf{3 1}$ & -2.425 & -2.48 & $\mathbf{3 2}$ & -4.85 & -2.48 & $\mathbf{3 3}$ & -6.998 & -1.24 \\
\hline $\mathbf{3 4}$ & -6.998 & 1.24 & $\mathbf{3 5}$ & -4.85 & 2.48 & $\mathbf{3 6}$ & -2.425 & 2.48 \\
\hline
\end{tabular}

This result shows that the value of the band-gap is quite good and establishes Pentacene to work as a semiconductor. There is another conversion of bandgap to wavelength to see whether the material is suitable for organic light-emitting diode material.

$\lambda=\frac{\mathrm{hc}}{\mathrm{E}}$

where $\mathrm{h}$ is the Planck's constant $\left(6.625 \times 10^{-34}\right)$ and $\mathrm{c}$ is the speed of light $\left(3 \times 10^{8} \mathrm{~m} / \mathrm{s}\right)$. Thus wavelength $(\lambda)$ for Pentacene here, by using this formula, is $895.629 \mathrm{~nm}$; these values is more than the visible range.

\subsection{Pentacene tuning with Nitro (Nitro-Pentacene)}

This first alteration to Pentacene was the introduction of the nitro $\left(\mathrm{NO}_{2}\right)$ substitute. The bond length between 
of the nitro $\left(\mathrm{NO}_{2}\right)$ substitute. The bond length between carbon is $1.4 \AA$ and is the same as with nitrogen to carbon, while nitrogen to oxygen bond length is 1.25 $\AA$ and with the same bond angle of $120^{\circ}$. Let's work with single Nitro; it can replace hydrogen at four different positions [28]. The combinations can be calculated by combination formula, given as:

${ }^{\mathrm{p}} \mathrm{C}_{\mathrm{r}}=\frac{\mathrm{p} !}{\mathrm{r} !(\mathrm{p}-\mathrm{r}) !}$

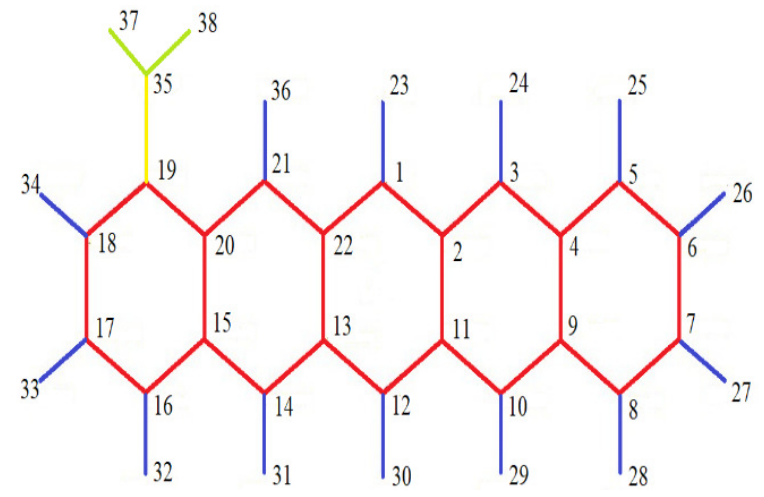

Fig. 5: 2-Nitro-Pentacene

where $\mathrm{p}$ is the number of places where nitro can be placed, $r$ is the number of nitro groups which in this case is 1 , so the result of this formula is 4 . This means that we can replace hydrogen with nitro at 4 different places. If we try to substitute nitro in any other place, we will get the same results as we get from these four combinations. This is because of the symmetry of the molecule. All these combinations were drawn on Auto Sketch version 15; one is shown in Fig. 5. Point 35 in
Fig. 5 is different from 25 of Fig. 4 i.e., $-4.85,2.48$. However, there are two new points 37,38 with coordinates $-5.933,3.425$ and $-3.767, \quad 3.425$ respectively. Results produced using nitro are ranged from 716 to $777 \mathrm{~nm}$. Only in two cases, the produced wavelength happened to be in the visible range.

\subsection{Pentacene tuning with other elements}

After that Pentacene was tuned by using single and double Fluorine, Chlorine, and Bromine. In the case of chlorine wavelengths produced were out of visible range [28] and hence was not promising. Whereas results produced using Fluorine and Bromine were quite promising and produces many visible shades. All the results along with the colors produced are shown in Table 3.

The substation was made using Fluoro-Pentacene, Bromide-Pentacene, and Nitro-Pentacene at all possible combinations that are $1,2,5$, and 6 . The results produced are shown in Fig. 6 as in graphical form and few in Table 4 with produced colors.

These computational results are very good to understand two things:

- The substitution does affect the bandgap and hence the wavelength.

- Change of substitution point also affects the bandgap.

The attempt of some more materials on Pentacene may produce better results. These results can be used for

\begin{tabular}{|c|c|c|c|c|c|c|}
\hline \multicolumn{6}{|c|}{ Table 4: Shade comparison of 2-Fluoro-Pentacene, 6-Nitro-Pentacene, and Bromide-Pentacene } \\
\hline Material & HOMO & LUMO & Hartee Energy & Bandgap(ev) & Wavelength(nm) & Color \\
\hline 1-Fluoro-Pentacene & 0.17061 & 0.10265 & 0.06796 & 1.84925956 & 671.722 & \\
\hline 6-Fluoro-Pentacene & 0.16946 & 0.10191 & 0.06755 & 1.83810305 & 675.799 & \\
\hline 2-Nitro-Pentacene & -0.1847 & -0.12099 & 0.0637 & 1.7327965 & 716.87 & \\
\hline 6-Nitro-Pentacene & -0.1883 & -0.1269 & 0.0615 & 1.67212 & 742.88 & \\
\hline 2-Bromide Pentacene & -0.2694 & -0.0978 & 0.1718 & -2.51756 & 493.4 & \\
\hline 1-Bromide Pentacene & -0.2732 & -0.1001 & 0.1731 & -2.26586 & 548.21 & \\
\hline 5-Bromide Pentacene & -0.2629 & -0.10001 & 0.1629 & -2.65905 & 467.15 & \\
\hline 6-Bromide Pentacene & -0.2742 & -0.10011 & 0.1741 & -2.38014 & 521.89 & \\
\hline
\end{tabular}




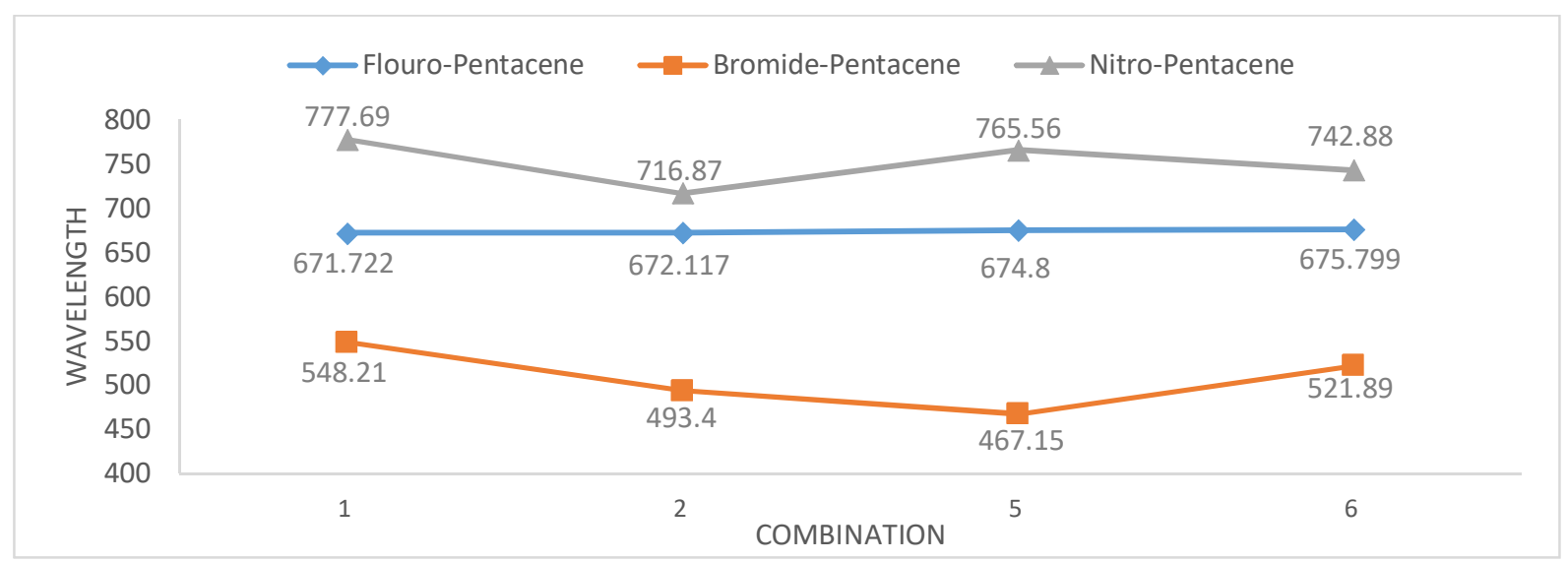

Fig. 6: Wavelengths (nm) of Flouro-Pentacene, Bromide-Pentacene, and Nitro-Pentacene Results at 1, 2,5 and 6 combinations.

the manufacturing purpose of the organic field-effect transistor, and hence can be used for organic displays.

By analyzing this Fig. 6, it can be concluded easily that compounds with strong negative nature emit small wavelengths if substituted at the corners of Pentacene as compared to the middle while the scenario is the other way around in case of positive nature substituent. The colors produced by 2-Fluoro-Pentacene, 6-NitroPentacene, and Bromide Pentacene are shown in table 4 where the difference in colors can be seen well. This result is a strong argument in defending the statement that by using different substituents, any color can be produced out of organic material. Hence organics materials can be used for the manufacturing of displays.

\section{CONCLUSION}

Results of substitution with fluorine, chlorine, bromine, and Nitro for Pentacene are good for this work. It was possible to produce some color out of them [31]. The substitution in the case of fluorine, chlorine, and bromine is electrophilic aromatic substitution (electron acceptor) while nucleophilic substitutions (electron donator) in the case of nitrate [28]. By changing the substitution point from corner to the middle of Pentacene, the wavelength in the case of electrophilic aromatic substitution decreases at the first point and keeps on increasing till the middle point while nucleophilic substitutions show exactly the reverse behavior. But the phenomenon doesn't apply in the case of bromine. So, this result is very important to understand the substitution effect. It is concluded that in most cases, by making an electrophilic aromatic substitution, we can expect more wavelength at the middle as compared to the point closer to the corners and vice versa in case of nucleophilic substitutions.

These results show that Pentacene is a good semiconductor for organic light-emitting diodes as its band-gap is within the range of semiconductor materials and all three basic colors can be produced out. Moreover, Pentacene can be substituted with many more materials to produce any desired shades.

\section{ACKNOWLEDGEMENT}

The authors wish to extend their gratitude to NSFC for sponsoring the research (No 61571189, 61871181) and to the State Administration of Foreign Experts Affairs for supporting the 111 project (ref: B13009).

\section{REFERENCES}

1. Memon B., Jatoi S., Ali Z., Larik J. R., Jamro L. A., "Improving Efficiency of Photovoltaic Cell Using Nanomaterials", Mehran University Research Journal of Engineering and Technology, Vol. 39, No. 1, pp. 55-62, Jan. 2020.

2. Neupane G. P., Ma W., Yildirim T., Tang Y., Zhang L., Lu Y., "2D organic semiconductors, the future of green nanotechnology", Nano Materials Science., Vol. 1, No. 4, pp. 246-259, December 2019. 
3. Lin L., Geng H., Shuai Z., Luo Y., "Theoretical insights into the charge transport in perylene diimides based n-type organic semiconductors," Organic Electronics, Vol. 13, No. 11, pp. 27632772, Nov. 2012.

4. Bratina G., Pavlica E., "Characterisation of charge carrier transport in thin organic semiconductor layers by time-of-flight photocurrent measurements", Organic Electronics, Vol. 64, pp. 117-130, January 2019.

5. Huang C.-Y., Wang J., Ding Z.-Y., Cui K., "Luminescent properties, internal hydrogen bonds and $\pi-\pi$ interactions of $\mathrm{Cd}(\mathrm{II}), \mathrm{Zn}(\mathrm{II})$, $\mathrm{Co}$ (II) complexes based on 2,8-di(pyridin-4yl)dibenzothiophene and dicarboxylate ligands", Journal of Molecular Structure, Vol. 1086, pp. 118-124, April 2015.

6. Esrafili M. D., Mohammadian-Sabet F., Solimannejad M., "Mutual influence between anion $-\pi$ and pnicogen bond interactions: The enhancement of $\mathrm{P} \cdots \mathrm{N}$ and $\mathrm{P} \cdots \mathrm{O}$ interactions by an anion- $\pi$ bond", Journal of Molecular Graphics and Modeling, Vol. 57, pp. 99-105, April 2015.

7. Sworakowski J., "How accurate are energies of HOMO and LUMO levels in small-molecule organic semiconductors determined from cyclic voltammetry or optical spectroscopy?", Synthetic Metals, Vol. 235, pp. 125-130, January 2018.

8. Venkatraman V., Steckl A. J., "Integrated OLED as excitation light source in fluorescent lateral flow immunoassays", Biosensors and Bioelectronics, Vol. 74, pp. 150-155, December 2015.

9. Xu L., Zheng G., Pei S., Wang J., "Investigation of optical bandgap variation and photoluminescence behavior in nanocrystalline $\mathrm{CuO}$ thin films," Optik (Stuttg)., Vol. 158, pp. 382-390, April 2018.

10. Colella M., Pander P., Monkman A. P., "Solution processable small molecule based TADF exciplex OLEDs", Organic Electronics, Vol. 62, pp. 168173, November 2018.

11. Cho D.-H., Kwon O.E., Park Y.-S., Yu B.-G., Lee J., Moon J., Cho H., Lee H., Cho N.S., "Flexible integrated OLED substrates prepared by printing and plating process", Organic Electronics, Vol. 50, pp. 170-176, November 2017.

12. Yu P., Zhen Y., Dong H., Hu W., "Crystal
Engineering of Organic Optoelectronic Materials," Chem, Vol. 5, No. 11, pp. 2814-2853, November 2019.

13. Hwang J., Sunesh C. D., Chandran M, Lee J., Choe Y., "Performance characteristics of pentacene-based organic photovoltaic cells," Organic Electronics, Vol. 13, no. 10, pp. 18091818, October 2012.

14. Chernov V., Alander J., Bochko V., "Integerbased accurate conversion between RGB and HSV color spaces," Journal of Electrical and Computer Engineering, Vol. 46, pp. 328-337, August 2015.

15. Mukherjee B., Kumar V.D., Gupta M., "A novel Hemispherical Dielectric Resonator Antenna on an Electromagnetic Band Gap substrate for broadband and high gain systems", AEU International Journal of Electronics and Communications, Vol. 68, No. 12, pp. 11851190, December 2014.

16. Prabu S., Nagalakshmi R., Balaji J., Srinivasan P., "Investigations on the vibrational modes and nonlinear optical properties of 4-Fluoro Chalcone crystal”, Spectrochimica Acta Part A: Molecular and Biomolecular Spectroscopy., Vol. 129, pp. 114-120, Aug. 2014.

17. Bassaco M. M., Monçalves M., Rinaldi F., Kaufman T. S., Silveira C. C., "Synthesis and photophysical characterization of novel $\pi$ conjugated vinyl sulfides," Journal of Photochemistry and Photobiology A: Chemistry, Vol. 290, pp. 1-10, September 2014.

18. Masai H., Terao J., Tsuji Y., "Insulated $\pi$ conjugated metallopolymers," Tetrahedron Letters, Vol. 55, No. 30, pp. 4035-4043, July 2014.

19. Mickens R. E., Herron I. H., "Approximate rational solutions to the Thomas-Fermi equation based on dynamic consistency", Applied. Mathematics Letters, Vol. 116, pp. 106-994, June 2021.

20. Santiago F.P., J. Mercado R.S., Brito B.M., "DFT/TD-DFT studies on electronic and photophysical properties of Auranofin: A reference $\mathrm{Au}(\mathrm{I})$ complex", Polyhedron, Vol. 11, pp. 42-62, December 2019.

21. Fu G. J., Johnson C. W., "From deformed Hartree-Fock to the nucleon-pair approximation", 
Physics Letters B, Vol. 809, October 2020.

22. Dwivedi A., Srivastava A. K., Bajpai A., "Vibrational spectra, HOMO, LUMO, MESP surfaces and reactivity descriptors of amylamine and its isomers: A DFT study", Spectrochimica Acta Part A" Molecular and Biomoecular. Spectroscopy, Vol. 149, pp. 343-351, October 2015.

23. Gonis A., "Generalization of the variational principle and the Hohenberg and Kohn theorems for excited states of Fermion systems", Physics Letters A, Vol. 381, No. 1, pp. 48-52, January 2017.

24. Englisch H., Englisch R., "Hohenberg-Kohn theorem and non-V-representable densities", Physica A: Statistical Mechanics and its Applications, Vol. 121, No. 1-2, pp. 253-268, August 1983.

25. Laeng B., Hugdahl K., Specht K., "The neural correlate of colour distances revealed with competing synaesthetic and real colours", Cortex, Vol. 47, No. 3, pp. 320-331, March 2011.

26. Skaff A.D., Miziorko H. M., “A visible wavelength spectrophotometric assay suitable for high-throughput screening of 3-hydroxy-3methylglutaryl-CoA synthase", Analytical Biochemistry, Vol. 396, No. 1, pp. 96-102, January 2010.
27. Khan M. A., Khan U. A., Ali A., Hussain F., Nisar W., "A Robust Color Image Watermarking Scheme using Chaos for Copyright Protection," Mehran University Research Journal of Engineering and Technology, Vol. 38, No. 2, pp. 361-378, April 2019.

28. Criquet J., Rodriguez E., Allard S., Wellauer S., Salhi E., Joll C., Gunten U.V., "Reaction of bromine and chlorine with phenolic compounds and natural organic matter extracts - Electrophilic aromatic substitution and oxidation," Water Research, Vol. 85, pp. 476-486, Nov. 2015.

29. Szpunar B., Szpunar J., "Density functional studies of selected metal dioxides", Journal of Physics and Chemistry of Solids, Vol. 74, No. 11, pp. 1632-1639, November 2013.

30. Borisov Y. A., Akhrem I. S., "DFT B3LYP/6$311+\mathrm{G}^{*}$ calculation study of a new nonclassical mechanism of electrophilic functionalization of aromatic $\mathrm{C}-\mathrm{H}$ bond via aryl cation formation", Mendeleev Communications, Vol. 29, No. 3, pp. 343-345, May 2019.

31. Park B., "Dielectric relaxation dependent memory elements in pentacene/[6,6]-phenyl-C61-butyric acid methyl ester bi-layer field effect transistors", Thin Solid Films, Vol. 578, pp. 156-160, March 2015. 